\title{
Capacidade de uso e manejo conservacionista do solo de um fragmento de cerrado sensu stricto, Montes Claros-MG
}

Capacity of use and soil conservation management of a fragment of cerrado sensu stricto, Montes Claros-MG

Capacidad de manejo de uso y conservación de suelos de un fragmento de cerrado sensu stricto, Montes Claros-MG

\section{Resumo}

A degradação do solo decorre de processos físicos e químicos. A atividade humana favorece sua aceleração por meio das práticas impróprias de manejo, como sobrepastejo intensivo e desmatamento. Estes processos implicam na redução do volume, fertilidade e atividade biológica do solo explorado, fazendo-se necessário o uso de práticas conservacionistas, o que aumenta a capacidade produtiva do solo e seu tempo de uso. Objetivou-se determinar a atual capacidade de uso do solo de uma área de cerrado sensu stricto. Para tais resultados, foram obtidos os dados referentes à clima, relevo, fertilidade do solo e histórico de uso da área. Com isso, a área foi classificada como imprópria para cultivos intensivos, mas com adaptação à pastagem. O solo possui problemas complexos de conservação, devido a pouca profundidade natural, o que foi agravado pela ação da erosão hídrica. A área foi classificada com declividade 
ligeira (5,37\%), o que pode acelerar a erosão hídrica. É recomendada a recomposição vegetal do solo, bem como o uso de curvas de nível.

Palavras-chave: Aptidão agrícola; Práticas conservacionistas; Erosão hídrica.

\begin{abstract}
Soil degradation results from physical and chemical processes. Human activity favors its acceleration through inappropriate management practices, such as intensive overgrazing and deforestation. These processes imply a reduction in the volume, fertility and biological activity of the exploited soil, making it necessary to use conservation practices, which increases the productive capacity of the soil and its time of use. The objective was to determine the current land use capacity of an area of cerrado sensu stricto. For such results, data referring to climate, relief, soil fertility and history of use of the area were obtained. As a result, the area was classified as unsuitable for intensive cultivation, but with adaptation to pasture. The soil has complex conservation problems, due to its shallow natural depth (5.37\%), which was aggravated by the action of water erosion. The area has been classified as having a slight slope, which can accelerate water erosion. The vegetal recomposition of the soil is recommended, as well as the use of contour lines.
\end{abstract}

Keywords: Agricultural aptitude; Conservation practices; Water erosion.

\title{
Resumen
}

La degradación del suelo es el resultado de procesos físicos y químicos. La actividad humana favorece su aceleración a través de prácticas de manejo inadecuadas, como el sobrepastoreo intensivo y la deforestación. Estos procesos implican una reducción del volumen, fertilidad y actividad biológica del suelo explotado, por lo que es necesario el uso de prácticas de conservación, lo que aumenta la capacidad productiva del suelo y su tiempo de uso. El objetivo fue determinar la capacidad actual de uso del suelo de un área de cerrado sensu stricto. Para tales resultados se obtuvieron datos referentes al clima, relieve, fertilidad del suelo e historial de uso del área. Como resultado, el área fue clasificada como no apta para cultivo intensivo, pero con adaptación a pastos. El suelo presenta complejos problemas de conservación, debido a su poca profundidad natural, que se vio agravada por la acción de la erosión hídrica. El área ha sido clasificada con una ligera pendiente (5.37\%), lo que puede acelerar la erosión hídrica. Se recomienda la recomposición vegetal del suelo, así como el uso de curvas de nivel.

Palabras clave: Aptitud agrícola; Prácticas de conservación; Erosión hídrica.

\section{Introdução}

O solo é um recurso natural multivalente e restrito, visto como um material solto e macio que cobre a superfície da terra (Moreira et al. 2013). Consiste no resultado da ação de fatores como material de origem, tipo de relevo e condições climáticas (Lepsch 2016). Adicionalmente, o autor defende que sua destruição pode acontecer por meio de eventos naturais como chuvas torrenciais ou pelo uso de práticas inadequadas.

Fatores físicos e químicos podem promover a degradação do potencial biológico do solo, interferindo em sua capacidade de sustentar a população a ela acoplada (Accioly 2010). A aceleração deste processo pode ser causada pela atividade humana por meio de práticas impróprias de manejo, pastejo intensivo e desmatamento (Sucupira 2006). Entretanto, processos naturais como chuvas e ventos podem auxiliar na formação de solos como também degradá-los pela erosão (Bertoni e Lombardi Neto 2005). Este processo consiste na retirada da camada superficial do solo, a sua parte mais produtiva, causando a redução dos níveis de matéria orgânica e de nutrientes, que por consequência acarreta a diminuição do potencial produtivo (Figueiredo et al. 2015).

O uso intensivo do solo, sem manejo adequado, resulta na sua compactação, diminuição do volume e estabilidade dos agregados, formando uma crosta superficial que diminui a infiltração, a penetração radicular, e consequentemente a cobertura vegetal (Manzatto et al. 2002). Tudo isso se intensifica com a erosão hídrica, que além disto, reduz o volume de solo, a disponibilidade de nutrientes e matéria orgânica deste, e sua atividade biológica (Pissara et al. 2005).

O solo predominante no cerrado é originado de espessas camadas de sedimentos que datam do Terciário. São solos profundos, azonados, de cor vermelha ou vermelha amarelada, porosos, permeáveis, bem drenados e devido a isto são intensamente lixiviados. A textura predominante é a fração areia, argila e por último o silte, arenosos, areno-argilosos, argiloarenosos com capacidade de retenção de água é relativamente baixa (Cunha et al. 2001; Coutinho 2000). 
Nesse contexto, as práticas conservacionistas ganham notoriedade, podendo serem a chave para alcançar um bom manejo de solo em determinada área (Silva et al. 2011). Tais práticas podem ser de caráter vegetativo, edáfico e mecânico; quando aplicadas corretamente podem garantir o uso do solo por muito mais tempo sem degradá-lo, e sem perder sua capacidade produtiva (Dedecek 1986). Assim, objetivou-se realizar um levantamento da capacidade de uso do solo e aptidão agrícola de terras para uma área de cerrado sensu stricto.

\section{Metodologia}

O fragmento (Figura 1) (0,58 ha) está localizado no Instituto de Ciências Agrárias da UFMG, Montes Claros-MG. O local possui cobertura vegetal rala; relatos de trabalhadores locais indicam histórico de corte profundo e seletivo, fogo intencional e pastejo intensivo. A vegetação do entorno é o cerrado sensu stricto (Figura 1, B-C). Conforme a classificação climática de Köppen, a região detém clima tropical - Aw. A precipitação média anual é de 1029 mm; o período seco se estende de abril a outubro. A temperatura média é de $22,7^{\circ} \mathrm{C}$; o mês mais quente é janeiro, que conta com uma temperatura média de $24,4^{\circ} \mathrm{C}$, e o mês mais frio é junho com uma média de $19,8^{\circ} \mathrm{C}$ (Figura 2) (INMET, 2021).

Figura 1. Fragmento de cerrado sensu stricto, Montes Claros-MG.

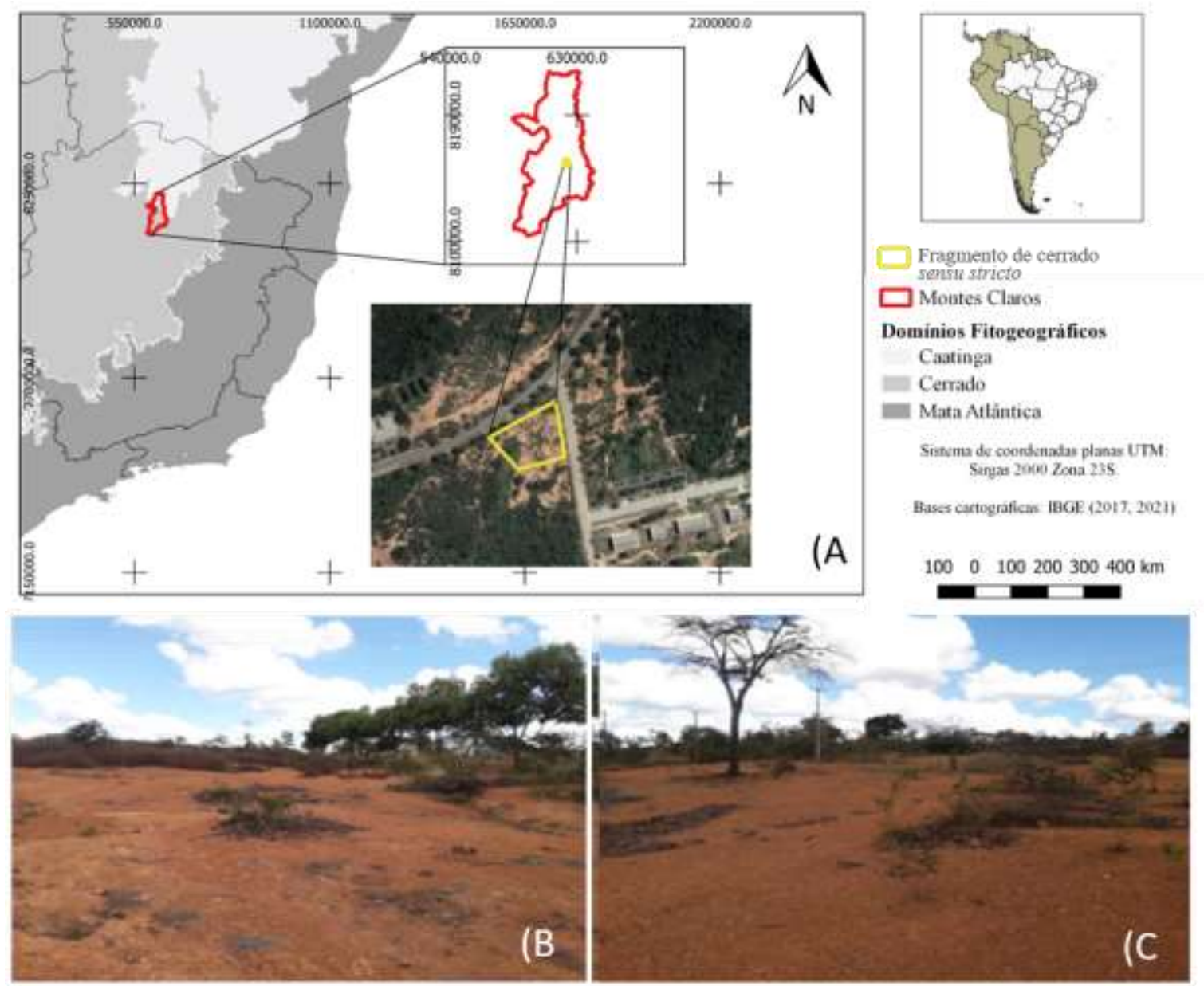

Legenda: A) Localização do cerrado sensu stricto estudado; B-C) Vista geral da área. Fonte: Autores. 
Figura 2. Dados climatológicos, Montes Claros-MG.

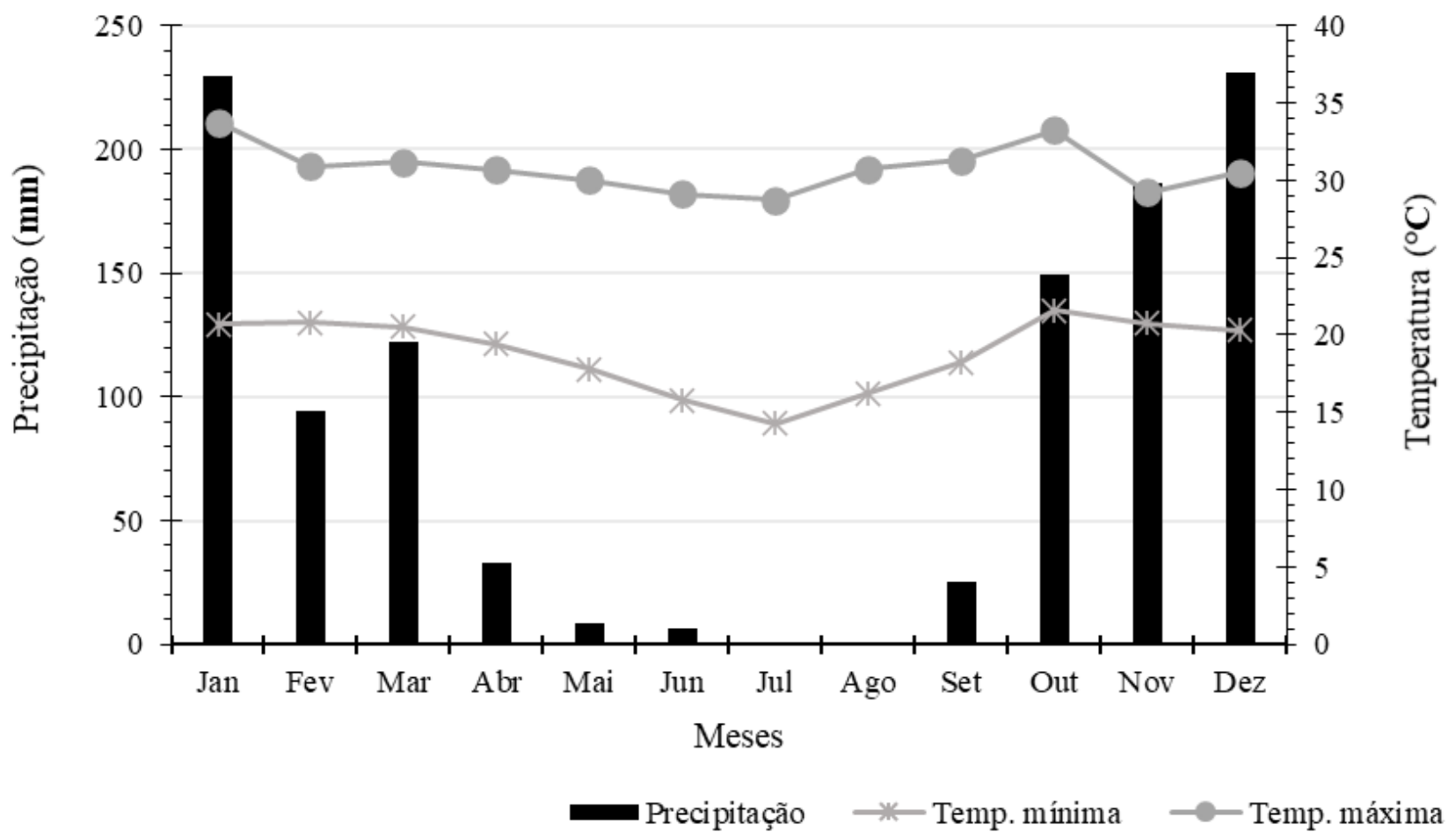

Fonte: Instituto Nacional de Meteorologia (INMET) (2021).

Foi realizada a análise de solo da área. Todas as espécies vegetais dentro da delimitação foram identificadas, classificadas quanto a origem (nativa do Brasil ou exótica) e hábito de vida. A declividade foi determinada por meio da razão entre medições de espaçamento vertical e horizontal entre dois pontos delimitados na área (método topográfico, por meio do nível de engenheiro). Os dados foram aplicados na seguinte fórmula:

$$
\begin{aligned}
& d / h=(s-i) /(b-c) \\
& d=(s-i) h /(b-c) \\
& h /(b-c)=k=100 \\
& d=100(s-i)
\end{aligned}
$$

Onde: $\mathrm{d}=$ declividade; $\mathrm{h}=$ distância do retículo ao centro focal; $\mathrm{s}=$ fio superior; $\mathrm{i}=$ fio inferior e $\mathrm{k}=$ credibilidade do solo.

Para classificação das terras adotou-se o Sistema de Classificação de Capacidade de Uso (Klingebiel e Montgomery, 1961), que possui as seguintes categorias:

Grupos (A, B e C), classes (de I a VIII), subclasses (e, s, a e c) e unidades (de 1 a 11).

GRUPO A - compreende as classes de I a IV (terras agrícolas);

GRUPO B - compreende as classes de V a VIII (pastagem e floresta);

GRUPO C - compreende a classe VIII (preservação).

A avaliação de aptidão agrícola seguiu o sistema de Classificação de Aptidão agrícola de terras (Ramalho Filho e 
Beek 1995), que descreve três níveis de manejo, seis grupos de aptidão agrícola e as suas respectivas classes:

Nível de Manejo A (primitivo)

Baseado em práticas agrícolas que refletem um baixo nível tecnológico, não há aplicação de capital para manejo, melhoramento e conservação das condições das terras e das lavouras, dependem do trabalho braçal, podendo ser utilizada alguma tração animal, com implementos agrícolas simples.

Nível de Manejo B (pouco desenvolvido)

Baseado em práticas agrícolas que refletem um nível tecnológico médio, modesta aplicação de capital e de resultados de pesquisas para manejo, melhoramento e conservação das condições das terras e das lavouras, condicionadas principalmente à tração animal.

Nível de Manejo C (desenvolvido)

Baseado em práticas agrícolas que refletem um alto nível tecnológico, aplicação intensiva de capital e de resultados de pesquisa para manejo, melhoramento e conservação das condições das terras e das lavouras, motomecanização presente nas diversas fases da operação agrícola.

Grupos de aptidão agrícola:

1. Aptidão boa para lavoura;

2. Aptidão regular para lavoura;

3. Aptidão restrita para lavoura;

4. Aptidão para pastagem plantada;

5. Aptidão para pastagem natural e silvicultura;

6. Inapto para qualquer tipo de exploração agrícola. Serve para refúgio de flora e fauna ou para fins de recreação.

Quanto à qualidade da aptidão agrícola e do manejo, foram consideradas as classes "ABC" Boa, "abc" Regular e (abc) Restrita, seguindo o modelo para "P" pastagens plantadas, "S" Silvicultura e "N" pastagens naturais.

Com base nas normais climatológicas (INMET 2021) (Figura 2) e análise de solo, foram calculados os parâmetros da equação universal de perda de solo.

Os fatores condicionantes da equação são:

$A=R \cdot K .(L . S) \cdot(C . P)(1)$

Onde: $\mathrm{A}$ = perda média anual de solo por unidade de área (ton/ha.ano); $\mathrm{R}=$ erosividade da chuva ( $\mathrm{Mj} . \mathrm{mm} / \mathrm{ha}$.h.ano); $\mathrm{K}=$ erodibilidade do solo $\mathrm{L}=$ comprimento da vertente; $\mathrm{S}=$ declividade da vertente; $\mathrm{C}=$ uso e manejo do solo; e $\mathrm{P}=$ práticas conservacionistas.

\section{Resultados e Discussão}

De acordo com os atributos químicos, o solo foi classificado como Cambissolo háplico eutrófico (Tabela 1). Os Cambissolos são caracterizados como solos de pouca profundidade e pouca permeabilidade, apresentando acidez pronunciada e, em muitos locais, sofrem severa erosão, por normalmente serem encontrados em áreas de declive ligeiro a moderado, necessitando de recuperação com práticas intensivas de conservação do solo (Sartori 2005). 
Tabela 1. Características químicas do solo.

\begin{tabular}{|c|c|c|}
\hline Determinação & Resultado & Nível \\
\hline pH em água & 5,4 & Baixo \\
\hline P Mehlich $\left(\mathrm{mg} \mathrm{dm}^{3}\right)$ & 0,15 & Muito baixo \\
\hline $\left.\mathrm{K}(\mathrm{mg} \mathrm{dm})^{3}\right)$ & 81 & Bom \\
\hline $\mathrm{Ca}\left(\mathrm{cmoic} \mathrm{dm}^{3}\right)$ & 3,5 & Bom \\
\hline $\mathrm{Mg}\left(\mathrm{cmoic} \mathrm{dm}^{3}\right)$ & 0,99 & Bom \\
\hline $\mathrm{Al}\left(\mathrm{cmoic} \mathrm{dm}^{3}\right)$ & 1,84 & Alto \\
\hline $\mathrm{H}+\mathrm{Al}\left(\mathrm{cmoic} \mathrm{dm}^{3}\right)$ & 2,05 & Baixo \\
\hline $\mathrm{SB}\left(\mathrm{cmoic} \mathrm{dm}^{3}\right)$ & 4,7 & Bom \\
\hline $\mathrm{t}\left(\mathrm{cmoic} \mathrm{dm}^{3}\right)$ & 6,54 & Bom \\
\hline $\mathrm{m}(\%)$ & 28 & Baixo \\
\hline $\mathrm{T}\left(\mathrm{cmoic} \mathrm{dm}^{3}\right)$ & 6,75 & Médio \\
\hline $\mathrm{V}(\%)$ & 70 & Bom \\
\hline MO (dag kg - 1) & 3,39 & Médio \\
\hline COT (dag kg - 1) & 1,96 & Médio \\
\hline
\end{tabular}

SB: Soma de bases; t: capacidade de troca catiônica efetiva; m: saturação por ácidos; T: capacidade de troca catiônica; V: saturação de bases; MO: matéria orgânica; COT: carbono orgânico total. Fonte: Autores.

Foram inventariados 10 táxons (Tabela 2). Todos bem distribuídos nas áreas do entorno, evidenciando que toleram as características naturais estressantes do local. Em relação à origem das espécies, foram classificadas como nativas (Flora do Brasil 2020). A presença de plantas nativas é um bom indicativo, pois, apesar do histórico com uso na agropecuária, o elevado número de sementes de espécies nativas se manteve viáveis e se desenvolveram na área. Em virtude de tolerarem as condições adversas extremas da área, como a erosão do solo (Lorenzi 1984), esses táxons também podem ser destacados como espécies chave para práticas de recuperação ambiental.

Em relação ao hábito, 3 das espécies são ervas, 1 é subarbusto, 2 são arbustos e 4 são árvores. A presença de ervas na área, demonstra sua importância, pois são consideradas pioneiras e quando necessárias, são capazes de iniciar a dinâmica sucessional em determinados ambientes (Costa et al. 2020). Fato que permite o estabelecimento de pioneiras e contribuindo com maior riqueza de espécies (Franco et al. 2012). 
Tabela 2. Espécies vegetais inventariadas.

\begin{tabular}{|c|c|c|c|}
\hline Família & Nome científico & hábito & Nome popular \\
\hline Anacardiaceae & Schinopsis brasiliensis & Árvore & Braúna \\
\hline Convolvulaceae & Evolvulus sp. & Erva & Azulzinha \\
\hline Fabaceae & Copaiba langsdorffii & Árvore & Copaíba \\
\hline Fabaceae & Chamaecrista flexuosa & Subarbusto & - \\
\hline Fabaceae & Mimosa pudica & Erva & Dormideira \\
\hline Malpighiaceae & Banisteriopsis gardeniana & Arbusto & Cipó-prata \\
\hline Malvaceae & Luehea divaricata & Árvore & Açoita-cavalo \\
\hline Malvaceae & Sida sp. & Erva & Vassoura \\
\hline Rubiaceae & Tocoyena formosa & Árvore & Genipapo-bravo \\
\hline Solanaceae & Solanum lycocarpum & Arbusto & Lobeira \\
\hline
\end{tabular}

Fonte: Autores.

O histórico de desmatamento desordenado, queimadas propositais e a intensa atividade de criação animal sem haver um manejo adequado respaldam a baixa riqueza vegetacional. Episódio que também alterou as características biológicas, químicas e físicas na área. Tal processo resultou na exposição direta do solo à ação das chuvas e dos ventos. Estas práticas descritas culminaram na perda do potencial produtivo da área e posterior processo erosivo.

A partir dos dados coletados em campo (Tabela 3) a área apresenta declividade de 5,37\% (Figura 3), caracterizando-a como ligeira. No fragmento não há curvas de nível definidas nem terraceamento, o que não interfere no uso de máquinas agrícolas. Entretanto, a ausência desta técnica de conservação pode acelerar alguns casos de erosão hídrica, fazendo-se necessário o uso de práticas de manejo conservacionistas. Além disso, a declividade do fragmento associada à falta de vegetação elevou o grau de erosão.

Tabela 3. Dados coletados em campo para medição de declividade.

\begin{tabular}{|c|c|c|c|}
\hline Fios & Sentido de menor declive & Fios & Sentido de maior declive \\
\hline Ponto inferior & 17 & $\mathrm{Pi}$ & 318 \\
\hline Ponto médio & 31 & $\mathrm{Pm}$ & 332 \\
\hline Ponto superior & 45 & $\mathrm{Ps}$ & 346 \\
\hline
\end{tabular}

Pi: ponto inferior; Pm: ponto médio; Ps: ponto superior. Fonte: Autores. 
Figura 3. Declive do fragmento.

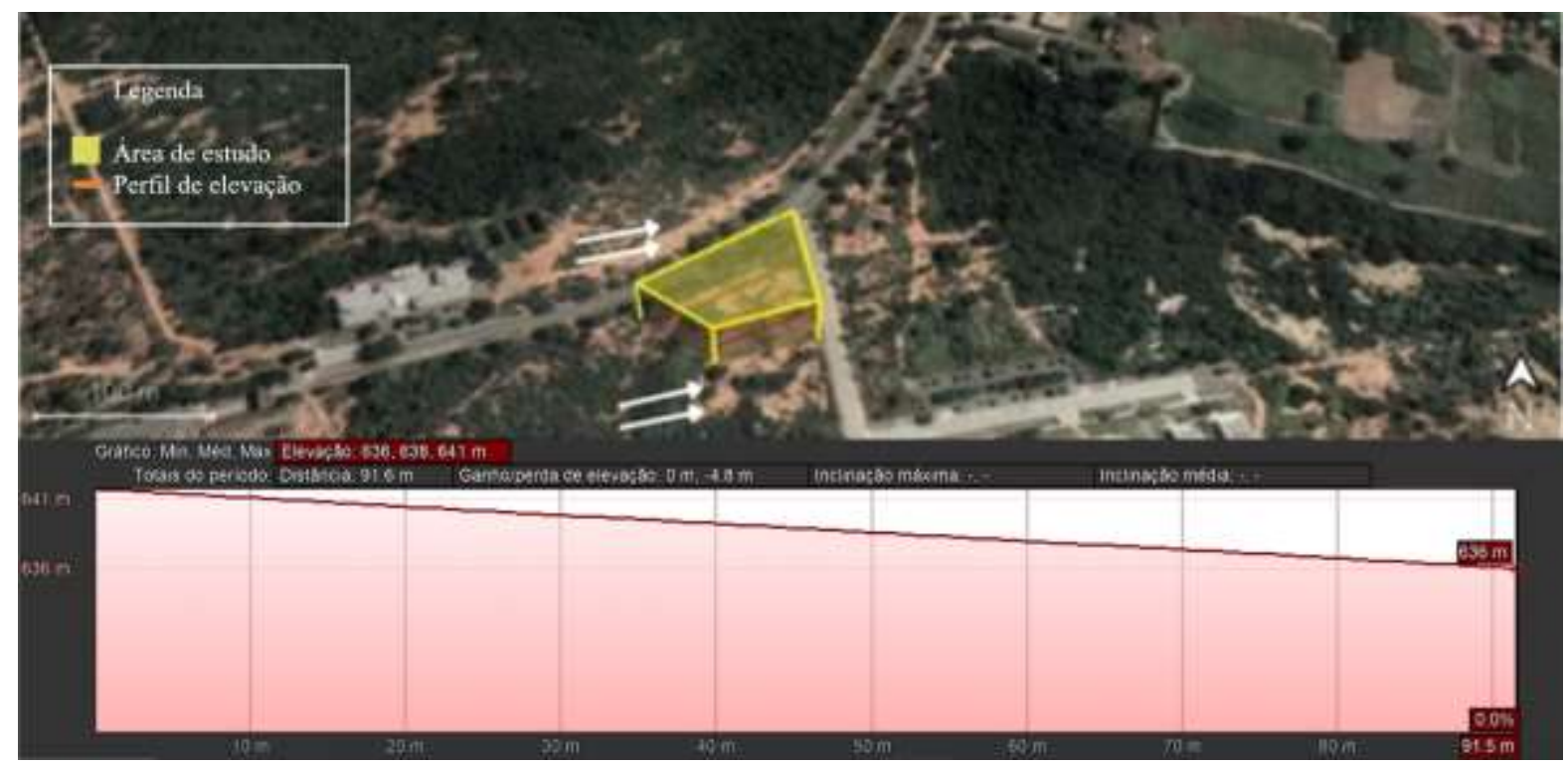

Fonte: Adaptado do software Google Earth (2021).

A capacidade de uso da área foi classificada como B, VIIs1,6, e2,4; caracterizando uma área imprópria para cultivos intensivos, porém é adaptada para pastagens. Possui problemas complexos de conservação do solo e da água devido à pouca profundidade e toxicidade por alumínio, o declive longo e as erosões laminar e em sulco. A aptidão agrícola foi 4 (p); Terras com aptidão regular para pastagem, o que confirma o potencial evidenciado na capacidade de uso.

Tal categoria de uso é proveniente da erosão laminar e em sulcos observadas na área, também originado por ações antropogênicas passadas. A erosão laminar é caracterizada pela junção de ações desagregadoras do impacto das chuvas (Santos et al. 2010) com o arraste de partículas de solo causado pelo escoamento superficial (Auerswald et al. 2009). Neste caso da área, houve arraste tanto de argila e matéria orgânica das camadas superficiais, quanto de nutrientes, sendo intensificada pelas ações antrópicas. Já a erosão em sulcos é resultado da concentração do escoamento superficial produzido por chuvas, uma vez que o impacto de suas gotas causa o desprendimento das partículas de solo, ocasionando a formação de pequenos canais (Polyakov e Nearing 2003). A erosão em sulcos é uma das mais agressivas (Govers e Rauws 1986), sendo extremamente severa em regiões onde as chuvas são intensas (Planchon et al. 1987), perfil observado na região, onde o período chuvoso se concentra em uma pequena parte do ano e com chuvas torrenciais. As erosões laminar e em sulco causaram grandes danos à área, pois reduziram a porção de solo e sua fertilidade, também decresceram e impossibilitaram o uso da terra para várias situações.

Com a Equação Universal de perda de Solo, foram obtidos os seguintes dados:

Comprimento de rampa: $56 \mathrm{~m}$;

Fator T (Tolerância): 5,52 t ha- ${ }^{1}$ ano;

Fator K (Erodibilidade): 0,054;

Fator R (Erosividade): $6.965,79 \mathrm{MJ} \mathrm{mm}^{-1}$ ha;

Fator LS (Topografia): 0,79.

A Equação Universal de Perdas de Solo é uma ferramenta de grande importância para estudo de processos erosivos em escala regional. Tal resultado embasou a categorização e diagnóstico a susceptibilidade natural do meio físico e vulnerabilidade à erosão da área. 


\section{Considerações Finais}

Para a área em estudo, é indicada intervenção mecânica para construção de curva de nível e posterior cobertura do solo, diminuindo o processo de erosão no qual a área é suscetível. Outros aspectos interessantes dessa tecnologia é o aproveitamento da água oriunda da chuva. Isso associado ao plantio direto mantém o nível de matéria orgânica constante e diminui a ação direta dos agentes erosivos quando o solo se encontra exposto.

Como opções de uso sustentável do solo, haja visto a área se encontrar em uma região semiárida, os sistemas agroflorestais têm emergido como alternativa, devido ao seu potencial de reduzir a degradação do solo e diminuir a pressão sobre as áreas de florestas. Estes se caracterizam pela combinação de espécies arbóreas com culturas agrícolas e/ou com animais domésticos, simultaneamente, ou alternados no tempo e no espaço. Essa prática vem contribuir para a redução da dependência de insumos externos, consistindo em uma prática viável para a exploração sustentável dos recursos de solos frágeis, como o Cambissolo.

Outra prática indicada é o emprego de cobertura vegetal herbácea a fim de proteger o terreno contra a erosão. Com a decomposição das raízes das plantas serão formados pequenos canais no solo, que aumentarão a infiltração da água. Além disso, haverá o melhoramento da estrutura do solo pela adição de matéria orgânica e, consequente, aumento da capacidade de retenção de água e a diminuição da velocidade de escoamento da enxurrada. Associado a isto, a cobertura vegetal servirá como proteção direta contra o impacto das gotas de chuva, que serão divididas em inúmeras gotículas, reduzindo sua força de impacto.

Instaurar pastagem natural ou plantada também são alternativas viáveis. Para tanto, a adubação química deve fazer parte do planejamento, onde devem ser considerados os custos e os benefícios desta atividade ao longo do tempo. Sua função é recompor os nutrientes perdidos pelo solo através de processos erosivos ou ainda de recuperação de solos inaptos para o cultivo. A manutenção da fertilidade é importante, pois proporciona melhor cobertura vegetal no terreno e consequente proteção do solo.

Os processos erosivos observados na área não foram tão significativos ao ponto de evidenciar uma situação crítica, entretanto dispor de práticas capazes de mitigar esses fatores são de extrema importância, principalmente pelo histórico de degradação da área. As práticas citadas acima (agroflorestas e pastagens), além de melhorarem consideravelmente os atributos do solo, contribuem para a geração de renda extra aos produtores, enfatizando os benefícios ambientais, sociais e econômicos da aplicação de técnicas de manejo e recuperação do solo.

\section{Agradecimentos}

Agradecemos a Professora Leidivan Almeida Frazão pelas análises de solo e uso dos equipamentos do laboratório sobre sua responsabilidade e ao Instituto de Ciências Agrárias da UFMG pelo apoio logístico e uso da estrutura física.

\section{Referências}

Accioly, L. J. de O. Degradação do solo e desertificação no Nordeste do Brasil. Embrapa Solos-Artigo de divulgação na mídia (INFOTECA-E), 2010.

Auerswald, K., Fiener, P., \& Dikau, R. (2009). Taxas de erosão de chapas e sulcos na Alemanha - Uma meta-análise. Geomorfologia, $111,182-193$.

(

Bertoni, J., \& Lombardi, N. F. Conservação do solo. Ícone, (1999). Conservação do solo. (5a ed.),

Costa, P. F. D., Pereira, Z. V., Fernandes, S. S. L., Fróes, C. Q., Santos, B. S. D., \& Barbosa, T. O. (2020). Banco de sementes do solo em áreas restauradas no sul do estado de Mato Grosso do Sul-MS. Ciência Florestal, 30(1), 104-116.

Coutinho, L. M. Aspectos do cerrado: solo. In: http://ecologia.ib.usp.br/cerrado/index.htm.

Cunha, T. J. F., Macedo, J. R., Ribeiro, L. P., Palmieri, F., Freitas, P. L. D., \& Aguiar, A. D. C. (2001). Impacto do manejo convencional sobre propriedades físicas e substâncias húmicas de solos sob cerrado. Ciência Rural, 31(1), 27-36. 
Research, Society and Development, v. 10, n. 7, e41410716697, 2021

(CC BY 4.0) | ISSN 2525-3409 | DOI: http://dx.doi.org/10.33448/rsd-v10i7.16697

da Silva, M. A., Silva, M. L. N., Curi, N., Avanzi, J. C., \& Leite, F. P. (2011). Sistemas de manejo em plantios florestais de eucalipto e perdas de solo e água na região do Vale do Rio Doce, MG. Ciência Florestal, 21(4), 765-776.

Dedecek, R. A. (1986). Erosão e práticas conservacionistas nos cerrados. Embrapa Cerrados-Circular Técnica (INFOTECA-E).

Figueiredo, T. D., Fonseca, F., \& Nunes, L. (2015). Proteção do solo e combate à desertificação: oportunidade para as regiões transfronteiriças. Proteção do solo e combate à desertificação: oportunidade para as regiões transfronteiriças.

Flora Do Brasil 2020. Jardim Botânico do Rio de Janeiro. In: http://floradobrasil.jbrj.gov.br/.

Franco, B. K. S., Martins, S. V., Faria, P. C. L., \& Ribeiro, G. A. (2012). Densidade e composição florística do banco de sementes de um trecho de floresta estacional semidecidual no campus da Universidade Federal de Viçosa, Revista Árvore, 36(3), 423-432.

Google (2020). Google Earth website. In: http://earth.google.com/.

Govers, G., \& Rauws, G. (1986). Transporting capacity of overland flow on plane and on irregular beds. Earth surface processes and landforms, 11(5), 515524.

Instituto Nacional de Meteorologia (INMET). In: http://www.inmet.gov.br/portal/Andgt.

Klingebiel, A. A., \& Montgomery, P. H. (1961). Land-capability classification (No. 210). Soil Conservation Service, US Department of Agriculture.

Lepsch, I. F. (2016). Formação e conservação dos solos. Oficina de textos.

Lorenzi, H. (1984). Manual de identificação e controle de plantas daninhas: plantio direto e convencional (No. 632.580981 L869). H. Lorenzi.

Manzatto, C. V., Freitas Junior, E. D., \& Peres, J. R. R. (2002). Uso agrícola dos solos brasileiros. Embrapa Solos.

Moreira, F. M. S., Cares, J. E., Zanetti, R., \& Sturmer, S. (2013). O ecossistema solo: componentes, relações ecológicas e efeitos na produção vegetal. Lavras: UFLA.

Pissarra, T. C., Galbiatti, J. A., Borges, M. J., Rosalen, D., \& Iha, D. (2005). Avaliação por fotointerpretação do uso/ocupação do solo e erosão acelerada em microbacias hidrográficas utilizando sistemas de informação geográfica. Simpósio Brasileiro de Sensoriamento Remoto, 12.

Planchon, O., Fritsch, E., Valentin, C. (1987). Rill development in a wet savanah environment. Catena. Supplement, (8), 55-70.

Polyakov, V. O., \& Nearing, M. A. (2003). Sediment transport in rill flow under deposition and detachment conditions. Catena, 51(1), 33-43.

Ramalho Filho, A., Beek, K. J. (1995) Sistema de avaliação da aptidão agrícola das terras.

Santos, G. G., Griebeler, N. P., \& de Oliveira, L. F. (2010). Chuvas intensas relacionadas à erosão hídrica. Revista Brasileira de Engenharia Agrícola e Ambiental, 14(2), 115-123.

Sartori, A., Lombardi Neto, F., \& Genovez, A. M. (2005). Classificação hidrológica de solos brasileiros para a estimativa da chuva excedente com o método do Serviço de Conservação do Solo dos Estados Unidos Parte 1: Classificação. Revista Brasileira de Recursos Hídricos, 10(4), 05-18.

Sucupira, P. A. P. (2006). Indicadores de degradação ambiental dos recursos hídricos superficiais no médio e baixo vale do rio Acaraú-CE. 2006.242 $p$ (Doctoral dissertation, Dissertação (Mestrado em Geografia) -Universidade Estadual do Ceará, Fortaleza). 